\title{
Experimental Investigation of Plastic Deformations Before Granular Avalanche
}

\author{
Axelle Amon.* Roman Bertoni, and Jérôme Crassous \\ Institut de Physique de Rennes, UMR UR1-CNRS 6251, \\ Université de Rennes 1, Campus de Beaulieu, F-35042 RENNES cedex, France
}

(Dated: Received: date / Revised version: date)

\begin{abstract}
We present an experimental study of the deformation inside a granular material that is progressively tilted. We investigate the deformation before the avalanche with a spatially resolved Diffusive Wave Spectroscopy setup. At the beginning of the inclination process, we first observe localized and isolated events in the bulk, with a density which decreases with the depth. As the angle of inclination increases, series of micro-failures occur periodically in the bulk, and finally a granular avalanche takes place. The micro-failures are observed only when the tilt angles are larger than a threshold angle much smaller than the granular avalanche angle. We have characterized the density of reorganizations and the localization of micro-failures. We have also explored the effect of the nature of the grains, the relative humidity conditions and the packing fraction of the sample. We discuss those observations in the framework of the plasticity of granular matter. Micro-failures may then be viewed as the result of the accumulation of numerous plastic events.
\end{abstract}

PACS numbers: 83.80.Fg,47.57.Gc,45.70.Ht

\section{INTRODUCTION}

The onset of avalanches in granular materials is a welldocumented area of research motivated by evident applications in the field of risk prevention. A typical granular experiment to study such phenomenon consists in the slow inclination of a sand filled box until the destabilization of the sand pile [1 $[3]$. A lot of studies have been devoted to the angles at which this avalanche initiates and stops [4, 5]. The critical angle, or avalanche angle, is then defined as the angle at which a large flow of grains occurs, with the surface of the heap stabilizing at a smaller angle, the angle of repose. Usually studies of stability of granular heaps focus on the succession of avalanches [1], either in rotating drums or by using a local perturbation of a heap. In those two cases, the heap has been built by successive avalanches, giving a specific internal structure to the bulk. The time intervals between successive events and the size of the avalanches is then discussed, one of the points being to know if those distributions are or are not power-law distributions as predicted for a critical phenomena [6]. In the case of a granular pile without initial inner structure progressively inclined in the gravitational field, specific types of rearrangements taking place before the first avalanche have been identified. Experimentally, two kinds of movement in the heap previous to its failure have been detected: small rearrangements, implying only a few grains, and periodic precursors of large amplitude [3, 7 [9]. Most of the studies reporting this phenomenology are based on the observation of the free surface of the pile, but recent works give clues that these precursors are bulk phenomena [7, 8]. To our knowledge, these regular events have never been reproduced numerically or explained theoretically, although preavalanche

*axelle.amon@univ-rennes1.fr instabilities showing an intermittent evolution have been studied numerically [10, 11].

The periodicity of the precursors are reminiscent of the stick-slip phenomenology, which is usually invoked as an explanation of the observed regularity. To our knowledge, a complete interpretation in that framework, including the prediction of the starting angle of the process, which is well beyond the angle of friction of the material, or of the selected period, has never been made. Stick-slip behavior emerges when a frictional system is submitted to an increasing load at a small enough rate 12]. It originates from the difference between the static friction and the dynamic friction. In the simplest model, a traction is exerted on a frictional slider by a spring. Elastic energy is first stored in the spring while the slider is stuck until the tangential force is large enough to overcome the static friction. The slider then enters an oscillating response that leads it to stick again when the velocity vanishes. A model inspired by this principle has been used to explain the succession of avalanches in a rotating drum [12]. In the same spirit, arrays of sliders connected by springs (Burridge-Knopoff model) [13] are used to model seismic processes. Fault gouges are often modeled in the geology literature by a granular material sheared between two planes and the stick-slip behavior of such systems has been extensively studied in an established regular regime of shearing of the granular layer [14, 15]. The existence of periodicity in seismology is still under discussion and recent models exhibit the coexistence of quasiperiodicity and criticality [16].

It is striking to observe that a very similar phenomenology of regular precursors of rupture before the establishment of the regular stick-slip response can be found in the literature in two other configurations closely linked to the problem of failure in granular materials: the onset of frictional motion and the mechanical response of metallic glasses. In the case of frictional motion, regular precursors are observed before the onset of sliding [17- 
20]. The present interpretation relies on the inhomogeneity of the spatial repartition of the normal and shear stresses at the interface leading to the possibility of reaching the Coulomb criteria in some localized areas before that the whole system is destabilized [21, 22]. The links with the models of earthquakes described in the previous paragraph have been made [23, 24]. In the second system, the case of the mechanical response of metallic glasses, the observed stick-slip phenomenology is called serration. The mechanical response of such amorphous glasses is very close to the response of granular material and displays similar features: creep, shear-banding and precursors to the rupture, all of which seem to be generic to amorphous materials. Comparing the loading curves obtained for metallic glasses (Figure 1(a) in 25]), granular materials (Figure 2(b) in 26]) and onset of friction (Figure 1(a) in 17]) a striking similarity is observed. Each of these three curves displays small regular stress drops during the otherwise quasi-elastic loading until the failure (or sliding) of the material. Those drops begin to appear well before the rupture of the material or the onset of motion of the slider. After the yield, the system enters a regular stick-slip motion. In the case of granular materials, it has been shown that the value of the stress at the first micro-rupture event is linked to the stress at which the global yield will take place [26]: independent of the packing fraction of the system, the ratio between those two stresses is constant. Using a method of detection identical to the one that will be used in this paper, it has been shown that the drops in the loading curve are linked to the appearance of a shear band in the system [27]. During the loading of the system, "spots" of localized strong deformation are also observed. As it will be shown, these localized spots tends to appear at a higher rate, and to cluster, just before a shear band event occurs [27].

Consequently, a natural question emerges regarding the understanding of the nature of those precursor events and their potential link to the small rearrangements. With this aim in mind, in this article, we study experimentally the response of granular material to a progressive, quasi-static, inclination. We will focus on the description of the behavior of the heap before the first avalanche. For the study of the rearrangements and of the precursors, we use an original method of measurement of small deformation based on Diffusive Wave Spectroscopy [28, 29]. This method gives access to the phenomenology of the rearrangements and the precursors at the side of the sample, and consequently throughout the depth of the pile, giving hints of what happens in the bulk of the system.

The structure of the article is as follows: in part $\coprod$ we will describe the mechanical setup ( $\amalg \mathrm{IA}$ ), the interferometric method of detection of the rearrangements and deformations ( $(\mathrm{IIB})$, and the granular materials and the experimental protocols of preparation of the samples ( 【C). In the third section we will present the observed experimental behavior. After an overview of the experimental observations ( the periodic precursor events (MIIB) and then the small rearrangements phenomenology (\$IIC). We also discuss the influence of several parameters (shape of the grains, humidity and packing fraction of the sample) on the observed phenomenology ( (IID). The results are discussed in $4 \mathrm{IV}$ In $\$ \mathrm{IVA}$ we compare the observations with the classical Mohr-Coulomb theory of failure. In IIVB, we interpret these events in the framework of plasticity of granular materials, and coupling between plastic events.

\section{EXPERIMENTAL SET-UP}

\section{A. Mechanical setup}

The mechanical set-up consists of an optical board that can be inclined continuously by a crankshaft system (see Fig. 1(a)). The laser source that illuminates the sample, the optical detection setup and the granular container are all fixed on the board so that the whole set-up rotates with the granular pile (see Fig. 1). The translation of the crankshaft is carried out by a motorised linear translation stage (Micro Controle UT100-50PP) controlled electronically (Micro Controle ITL09). This system ensures a low level of vibration at a small rotation rate. For translations between $25 \mu \mathrm{m} / \mathrm{s}$ and $100 \mu \mathrm{m} / \mathrm{s}$, the tilt rates range between $0.02^{\circ} / \mathrm{s}$ and $0.08^{\circ} / \mathrm{s}$. We will show in the following that all the experiments can be considered to be done in a quasi-static limit.
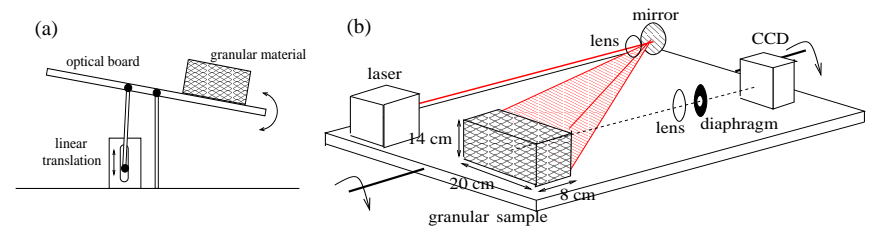

FIG. 1. (a) Schematic of the crankshaft system with the tilting optical board. (b) Schematic of the setup clamped to the tilting optical table. The laser source is expanded in order to illuminate the side of the sample. The backscattered light is collected on a CCD camera. A lens images the side of the sample on the CCD and a diaphragm controls the size of the speckles on the camera.

The granular sample is contained in a rectangular box of dimensions $20 \mathrm{~cm} \times 14 \mathrm{~cm} \times 8 \mathrm{~cm}$, the lateral sides of which are glass windows. One lateral side is illuminated by a laser (Melles Griot, $15 \mathrm{~mW}, 633 \mathrm{~nm}$ ). The beam of the laser is expanded by a lens in order to illuminate the entire surface of one side of the box. A lens images that side of the sample on a CCD camera (Prosilica GC $2450,2448 \times 2050$ pixels, cell size $3.45 \times 3.45 \mu \mathrm{m}$ ), with a magnification $\gamma=0.09$. Because of the disorder of the granular media, the backscattered light collected has a speckle pattern in intensity. A diaphragm allows us to adjust the size of these speckles on the CCD camera (see next section for the optical method). The camera 
is interfaced with a computer and a Labview program ensures the acquisition of successive images at a frame rate that has been chosen between $1 \mathrm{fps}$ and $3 \mathrm{fps}$ in the experiments shown here.

\section{B. Optical method}

The measurement of deformation and rearrangements in the granular material is carried out using an interferometric technique based on Diffusive Wave Spectroscopy (DWS). The method has been extensively described in [28, 29]. The treatment of raw experimental images is based on the correlation of backscattered intensity between two successive images following a multispeckle procedure. The link with the corresponding deformations that have occurred inside the sample is made using a model [28, 30].

The schematic of the optical part of the setup is shown in Figure 1(b). The beam of a laser source is expanded to illuminate the granular sample and the side of the sample is imaged on a camera with a lens. Because of the disordered structure of the scattering material, light rays inside the media follow random paths. The backscattered rays explore a typical zone of length $l^{*}$ in the sample, called the transport mean free path of light. For glass spheres of diameter $d, l^{*} \simeq 3.3 d[30$. As the incident light is coherent, those backscattered rays interfere and give a speckled structure to the collected intensity. The size of the coherence areas of the speckle in the image plane is controlled with a diaphragm. When the sample is deformed, the scatterers are displaced and the light paths modified so that the interference pattern changes. These changes can be quantified by calculating the correlation of intensities between two states.

In a backscattering configuration, a light ray typically explores a volume of characteristic size $\left(l^{*}\right)^{3}$ inside the scattering material. By using domains of size $\left(\gamma l^{*}\right)^{2}$ in the images for the ensemble average, we can then obtain a local evaluation of the correlation function with the optimal spatial resolution. Correlation maps are thus obtained, giving insights into the processes that have taken place in the sample during the loading.

The intensity correlation between two successive images, labelled 1 and 2 , is calculated using the relation:

$$
G_{I}=\frac{\left\langle I_{1} I_{2}\right\rangle-\left\langle I_{1}\right\rangle\left\langle I_{2}\right\rangle}{\sqrt{\left\langle I_{1}^{2}\right\rangle-\left\langle I_{1}\right\rangle^{2}} \sqrt{\left\langle I_{2}^{2}\right\rangle-\left\langle I_{2}\right\rangle^{2}}},
$$

where $I_{1}$ and $I_{2}$ are the scattered intensities on images 1 and 2. The electronic noise of the camera and possible stray light are taken into account with a procedure explained in [31]. The ensemble averages $\langle\cdot\rangle$ are calculated over square sub-areas of the images of size $16 \times 16$ pixels, which typically contain about forty coherence areas. From initial images of size $2448 \times 2050$ pixels we then obtain maps of $153 \times 128$ pixels. With the lens magnification chosen here, the spatial resolution of the final map is about $608 \mu \mathrm{m}$.
The map of correlations are calculated between successive images, giving information about the incremental decorrelations that have taken place in the system for an angle increment. When the tilt rate was modified, the frame rate of the camera was chosen to ensure that the angle increment between two successive images stayed between $0.06^{\circ} /$ slice and $0.08^{\circ} /$ slice. This allowed for consistency and ease of comparison between the different experiments.

In the case of linear elastic samples 28], the deformation of the material corresponding to the measured decorrelation can be estimated using a model. The extension of that model in the case of granular materials has been discussed in [29]. Considering that the deformation between the two correlated images is affine at the scale of $l^{*}$ in the material, that the light is strongly scattered in the sample, and that we observe the backscattered light, the expected dependence of the intensity correlation function on the strain tensor is as follows:

$$
G_{I} \simeq \exp (-c \sqrt{f(\mathbf{U})}),
$$

where $f(\mathbf{U})$ is a quadratic function of the strain tensor $\mathbf{U}$, (see 28, 29] for details of that function), corresponding to the deformation increment that has taken place between the two states associated with the images used for the calculation of the correlation [28, 29]. Following [29], the constant $c$ has been taken as $c=2 \eta \frac{2 \pi}{\lambda} l^{*} \sqrt{\frac{2}{5}}$, with $\eta \simeq 2$ a constant depending of the optical setup and $\lambda$ the wavelength of the laser. The typical deformations probed by this method for values of the transport length similar to the one of the glass beads used here, $l^{*} \simeq$ $990 \mu \mathrm{m}$, are between $10^{-6}$ and $10^{-4}$ [27, 29]. For sand, we measured $l^{*} \simeq 660 \mu \mathrm{m} \simeq 2 d$. This lower value of $l^{*} / d$ compared to glass beads may be understood by noticing that the surface of sand grains should itself diffuse light. However, since the ratio $l^{*} / d$ is not very different for glass beads and sand, we expect the magnitude of probed deformations to be very similar.

\section{Granular samples and preparation}

Experiments have been performed for two different type of grains: glass beads (Silibeads, Figure 2(a)) and sieved sand (Sifraco, Figure 2(b)) with a similar range of diameters: $200-400 \mu \mathrm{m}$ as can been seen on the cumulative size distributions for the two materials in Figure 2 . We observe in the insert pictures in Figure 2 that the glass beads have a lot of defects and that the main difference between the two kind of grains is that the sand is faceted whereas the glass beads are rather spherical. The angle of avalanche for the glass beads is about $29^{\circ}$ and for sand about $35.5^{\circ}$. The cumulative distributions show the presence of few particles in the range 50-100 $\mu \mathrm{m}$ for the two types of granular material. Since the dynamics of precursors is in agreement with previously reported experiments with larger beads [3, 7,9$]$, we may think that 
the details of the particle size distribution is not crucial.
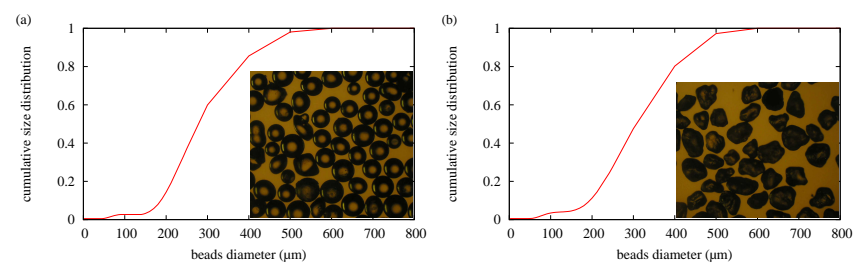

FIG. 2. Cumulative distribution of the grain diameter for the glass beads (a) and the sand (b). Pictures of the granular materials are shown in inserts. The median size of the grains is about $280 \mu \mathrm{m}$ for the glass beads and $330 \mu \mathrm{m}$ for the sand.

Three different levels of compaction have been used. For the lowest compaction samples, a grid (mesh size $4 \mathrm{~mm}$ ) is placed at the bottom of the empty box, the granular material is gently poured in the box, and then the grid is slowly lifted through the sample. More compacted configurations are obtained from this initial loose preparation by tapping the filled box in order to increase the density of the system. Grids of different mesh sizes and forms have been tested not to change the global phenomenology. The less compacted samples have packing fraction around $C \simeq .58 \pm .02$, the intermediate case (called "normal" in the following) has $C \simeq .61 \pm .02$, and the highest packing fraction is close to $C \simeq .64$.

Four different relative humidity conditions have been used. Ambient humidity was between $25 \%$ and $45 \%$ (called "normal" condition in the following). We obtain a humidity smaller than $20 \%$ by leaving the granular sample in an oven overnight and then letting it cool down in presence of desiccant. We obtain high humidity by leaving the granular sample overnight in an enclosure in the presence of either saturated salt water or pure water. The relative humidity in the enclosure is controlled by the nature of the salt, and we then obtain samples prepared at respectively $70 \%$ and above $80 \%$ humidity. For all those values of the relative humidity, the dynamics is unchanged when modifying the duration of the experiment. So we can neglect relative humidity variations at the timescale of the experiment.

\section{EXPERIMENTAL RESULTS}

\section{A. Main phenomenology}

A typical movie of the observed behavior in the socalled "normal" conditions (packing fraction around .61 and relative humidity of $25 \%$ ) is given in Supplemental Material [32]. The granular material used in this movie is glass beads but the phenomenology is very similar to sand. Each image is a map of the correlation between two successive images and corresponds to the incremental deformation during an angle variation of $0.08^{\circ}$. The color scale is the following: white correspond to $G_{I}=1$ (maximal correlation) and black to $G_{I}=0$ (vanishing correlation). The value of the angle of inclination of the board is indicated in degrees at the bottom-left of the film. The free surface of the granular pile can clearly be identified due to the fact that the light that does not come from the sample is totally decorrelated. The size of the area seen in the film is $7 \mathrm{~cm} \times 8.5 \mathrm{~cm}$ from the upper middle of the box (see the schematic in Figure 3). In order to evidence the small rearrangements, the contrast of the maps shown on Figure 3 has been enhanced using a threshold value of $G_{I}=0.75$, so that all the correlations smaller than that value appear in black.

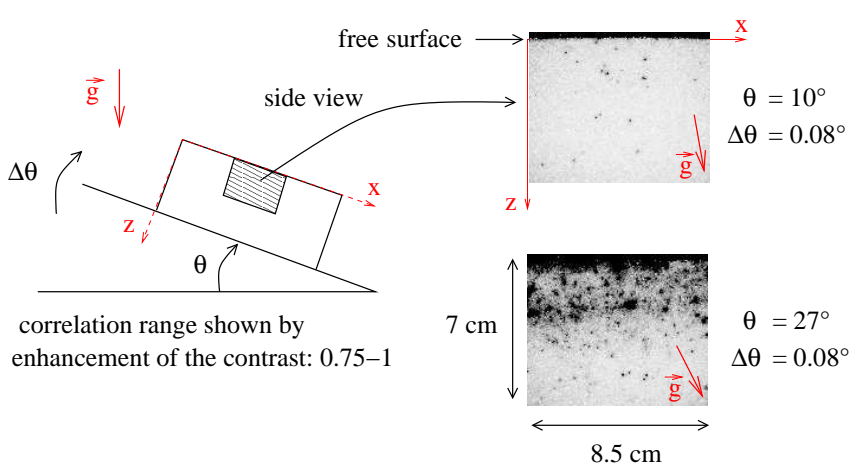

FIG. 3. Schematic of the area used in the movie in Supplemental Material [32]. Two examples of correlation maps extracted from the film, for angles $10^{\circ}$ and $27^{\circ}$, are shown and exhibit typical rearrangements. The decorrelation maps of these images have been thresholded for $G_{I}$ smaller that 0.75 to allow the rearrangements to be clearly identified. The granular material is glass beads, the packing fraction around .61 and hygrometry of $25 \%$. The convention used for the axes are indicated on the figure.

Two distinct types of phenomenology can be observed. Firstly, spots of different sizes appear well before any macroscopic event in the sample. Such events are shown for example on Figure 3 for the $10^{\circ}$ angle. Enhancement of the contrast shows that such events appear as soon as the inclination process begins. These spots are seemingly of the same nature as the rearrangements described previously in the literature [3] and which were observed at the free surface of the sample. Our observation tends to show that those rearrangements happen in the whole bulk and are not mere displacements of grains due to irregularities of the free surface originating from the preparation of the sample, as has been postulated in previous studies. The density and the intensity of those events increase with the angle. The density seems also to depend on the depth in the sample. Such localised events are reminiscent of the spots observed in a shear granular sample in 27]. The fact that such spots have been observed at different borders in several systems limited by different boundary conditions supports the hypothesis that such localized events happen in the bulk of the sample. A quantitative estimation of the energy released locally compared to the overall work done during creep processes [27] also supports that affirmation. The spots were identified in [27] as the localized events of deforma- 
tion introduced by Argon [33] to describe the plasticity of amorphous materials.

Secondly, from an angle of about $15^{\circ}$, large and almost regular events begin to happen, which appear as successive large decorrelation zones. These events correspond to the periodic precursors already described in the literature [3]. Our experiment makes it evident that these precursors involve a large part of the bulk. These precursors begin to happen from about $15^{\circ}$ and occur nearly regularly, with an angular periodicity between $1^{\circ}$ and $2^{\circ}$. Quantitatively, these values correspond well to the values that have been reported in the literature for other sizes of grains and containers $[3,7,9]$. We can observe that a precursor mobilizes a slice of the sample parallel to the free surface. Such precursors are in fact micro-rupture events in the bulk material. We also observe that the position of the micro-rupture occurs at a depth that increases with each consecutive event. Between two precursors, local rearrangements are also still observed. Because of the approximative translational invariance of the phenomenon along the optical plane, we obtain a spatiotemporal representation by averaging the correlations at constant depth in each image along horizontal lines $(x-$ axis): $\left\langle G_{I}(\theta ; \theta+\Delta \theta ; x ; z)\right\rangle_{x}$. This gives a $1 \mathrm{D}$ representation of the dependence of the correlation with depth for each image. By juxtaposing these 1D lines for successive angles of inclination, we obtain spatio-temporal graphs such as the one shown on Figure 4. As the dependence between time and angle is linear in a good approximation, the horizontal axis can be considered as representing either time or angle. On such a representation the periodic precursors are displayed distinctively while the rearrangements contribute to an average decrease of the correlation, giving a blurred or shaded aspect to the image where the activity in terms of small rearrangements is high.

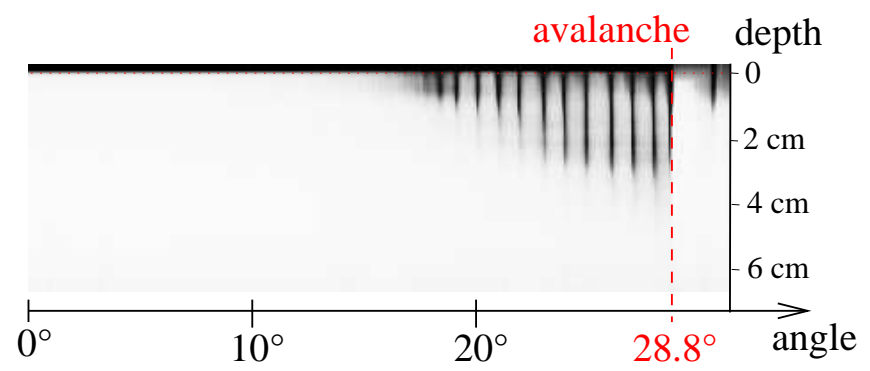

FIG. 4. Spatiotemporal diagram obtained from the correlations map by spatially averaging each horizontal line of the images: $\left\langle G_{I}(\theta ; \theta+\Delta \theta ; x ; z)\right\rangle_{x}$. The averaging procedure gives the mean decorrelation as a function of depth for each image. The granular material is glass beads, the packing fraction around .61 and relative humidity of $25 \%$. Correlations are calculated between images acquired at angles $\theta$ and $\theta+\Delta \theta$, with $\Delta \theta=0.08^{\circ}$.

It is noticeable in the movie or in Figure 3 at the angle of $27^{\circ}$ that before and after a micro-rupture event occurs, a large amount of activity can be observed at the depth where the failure will take place. Larger spots of decorrelation are observed in the zone that will fail compared to those that appear at a larger depth. The decorrelation events seem to align and cluster at the place where the rupture will take place, reminiscent of the phenomenology already observed in a sheared granular material [26, 27] where regular micro-ruptures were also observed before the final yield stress of the material is attained.

In the following section we give a more detailed study of that global phenomenology which is very robust and resistant to changes of parameters (grain shape, relative humidity, packing fraction). Firstly we will detail the precursors events, then we will describe the rearrangements. Finally, we will study the changes of the phenomenology with hygrometry and packing fraction.

\section{B. Precursors}

\section{Periodicity}

We have studied experimentally the dependence of the periodicity with respect to the tilt velocity. Figure 5 shows the average period of the precursors in degrees for different rotation rates for the same conditions of relative humidity and packing fraction (so-called "normal" conditions: packing fraction about 0.61 and relative humidity about $30 \%$ ). Each point corresponds to the average period between the precursors during one run. The value of the period slightly decreases when the tilt rate increases, but overall the phenomenon seems not to depend on the tilt rate. This mean that the experiments can be considered as having been carried out in the quasi-static limit. We also see in Figure 5 that the nature of the material, glass beads (green (light gray) points) or sand (black triangles), does not seem to modify significantly the overall mean value of the period which is a little over $1^{\circ}$ for both materials. The error bars give the minimal and maximal values of the period during each run. That dispersion around the mean value follows a trend during one experiment: a significative increase of the period, given by the size of the error bars, has been observed between the first occurrence of the precursors around $15^{\circ}$ and the avalanche angle.

To conclude, the periodicity does not depend of the tilt rate in the quasi-static limit for which the experiments have been done. It is therefore justifiable to express that periodicity in terms of angles and not in terms of time intervals. The period has been found to be about $1^{\circ}$ for the two types of granular materials. It has to be noted that similar values of the period have been obtained for much larger beads (2 to $3 \mathrm{~mm}$ ), in containers of different sizes and materials [3, 7,9$]$. 


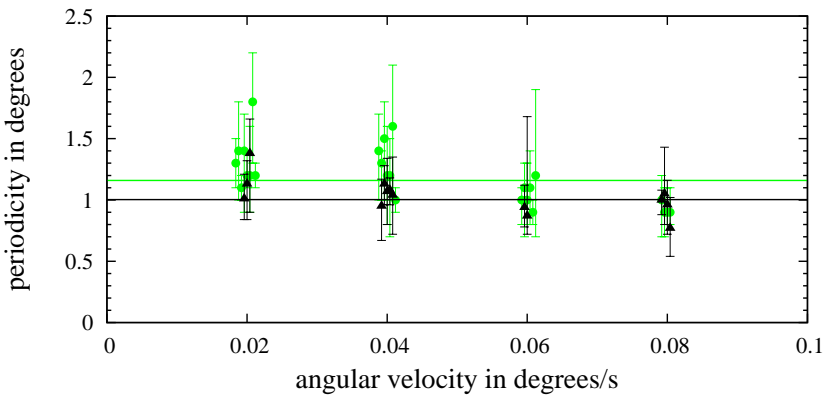

FIG. 5. (Color online) Average period of the precursors with the tilt rate of the sample. The error bars give the extremal values of the period around the mean value during one experiment. Green (light gray) points are for glass beads, black triangles for sand. The horizontal lines give the average value of the period over all the runs for each type of material. All the experiments have been done with a similar preparation: packing fraction about 0.61 and relative humidity about $30 \%$

\section{Size of the precursors}

An important question that remained unanswered in previous works was whether the bulk is involved in the rearrangements and precursors phenomena. As a matter of fact, most of the previous experiments have been carried out using only free surface observations [3, 9], so that rearrangements and precursors could be supposed to be a mere free surface phenomenon. On the other hand, acoustic measurements [7, 8] gave rather indirect indications of the bulk mobilization. In our experiment we are able to visualize directly how deep the phenomena go inside the sample, even if the observation is confined to a thin layer near the wall of the container. We show that our results reinforce the hypothesis that a part of the bulk is indeed mobilized in a precursor event. The volume that is involved increases as the system comes closer to the avalanche angle.

The successive depths of precursors can be obtained from the spatiotemporal graphs such as the one of Figure 4. The values of the depth of the peaks, normalized by the diameter of the grains, as a function of the angle at which they appear are shown on Figure 6. The main part of the graph shows the evolution of those depths for different typical experiments. We can observe that the dependence of the size of the precursors with the inclination angle is roughly linear, even if in some experiments brutal "jumps" can be observed (results displayed with solid lines). The green (light gray) lines correspond to experiments with glass beads and the black lines to the ones with sand. The slope of increase of depth with the angle is generally larger for the glass beads than for the sand. Also, the first precursor tends to appear sooner for glass beads than for sand. The insert of Figure 6 shows all the results from 25 experiments for glass beads and 14 for sand superimposed, giving an idea of the dispersion of the results from one experiment to the other. All the ex-

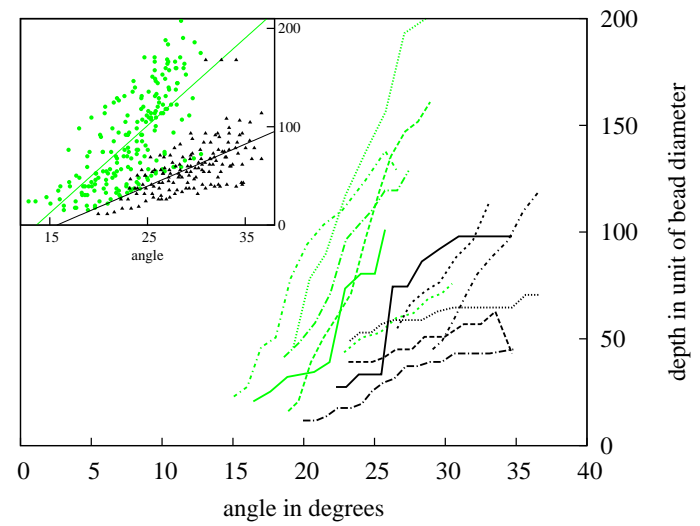

FIG. 6. (Color online) Depth $z$, in units of grain diameter $d$, of the successive precursors plotted as a function of the angle of inclination of the granular sample $\theta$. All the experiments have been done for "normal" compaction $(\sim .61)$ and relative humidity conditions $(\sim 30-40 \%)$ but different tilt rates. Main graph: examples of evolution for glass beads (green (light gray) lines) and sand (black lines) showing the roughly linear dependence of the depth with the angle. Insert: gathered results for a total of 25 experiments for glass beads (green (light gray) points) and 14 experiments for sand (black triangles). Lines are linear fits of those data: for glass beads, $z / d=9 \times\left(\theta-14^{\circ}\right)$, for sand: $z / d=4 \times\left(\theta-16^{\circ}\right)$.

periments have been done in the "normal" conditions of relative humidity and packing fraction and for tilt rates between $0.02^{\circ} / \mathrm{s}$ and $0.08^{\circ} / \mathrm{s}$. Linear fits using the whole set of data for each type of granular material have been done and are shown as solid lines in the insert. The slope of the line is about 9 beads diameter per degree for the glass beads and 4 for the sand. For the two types of material, the intersection of the linear fit with the abscissa axis is about $15^{\circ}$, even if the first precursors appear later in sand than in glass beads.

Thus, precursor events involve a fraction of the material that increases roughly linearly with the angle of inclination. That increase is approximatively two times larger for the glass beads than for the sand. The part of the sample that is mobilized is parallel to the surface, such that the process seems to imply a rectangular block of granular material in the upper part of the sample. A closer observation of the movie in Supplemental Material 32] shows that the surface of separation between the mobilized zone and the quiescent zone bends toward the bottom of the box, which could be an effect of the side boundaries. A more detailed description of the processes happening in the decorrelated area during a precursor event is difficult giving the impossibility of separating the contributions from deformation, plastic rearrangements and large scale displacement of the block to the decorrelation. Nevertheless, some hints of the process can be inferred from a sequence before a micro-rupture, as shown in Figure 7 We can see on this figure a "rupture" occurring at an angle of $22.32^{\circ}$. Yet the failure happens before as evidenced by a decorrelated zone mainly 


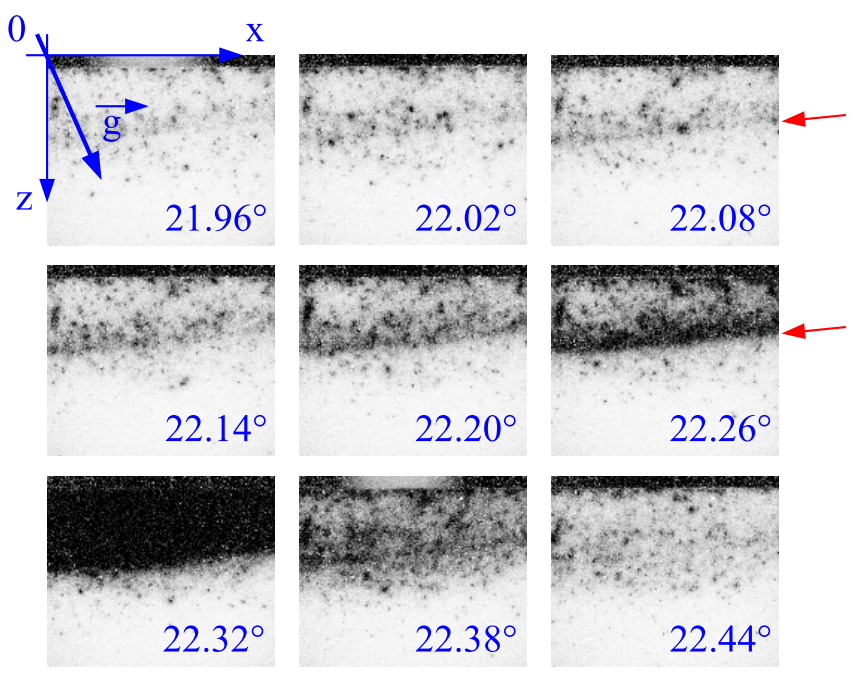

FIG. 7. Detailed sequence describing a micro-slip event for an experiment with glass beads at "normal" hygrometry and packing fraction condition. The images shown are consecutive for angles around $22^{\circ}$. The angle increment between the states used to calculate each correlation map is $0.06^{\circ}$. The red arrows show the localisation of the failure plane.

along a line (see arrow on fig. $722.26^{\circ}$ ). This line is indicative of a zone of shearing between two translating blocks. As a matter of fact, a small solid translation does not modify the optical paths relative to each other and the images stay correlated. Nevertheless, the two blocks are not mechanically identical: the activity in the upper section is clearly larger than in the bottom. Finally, the micro-rupture event occurs. It is not temporally resolved and appears as a large totally decorrelated area. Please note that the total time of the sequence is $\sim 3 \mathrm{~s}$, which is very large compared to the inertial time corresponding to a displacement of, say, one bead diameter $\sqrt{d / g} \simeq 5 \mathrm{~ms}$. So, inertial effects do not seem to play a role in the development of the micro-rupture. The phenomenology stays globally the same from one experiment to the other, either for sand or glass beads: the density of rearrangements increases in the area where the rupture will take place, nevertheless a line of decorrelation at some depth of the sample as the one distinctly seen at $22.26^{\circ}$ on Figure 7 is not always clearly seen.

We thus observe that the density of rearrangements and the large periodic precursors events are not independent. Consequently, in the following section we will focus on the quantitative study of the rearrangements in the sample.

\section{Analysis of the rearrangements}

\section{Density of activity in the material}

To evidence the level of activity in the samples during the inclination process, we first threshold the spatio- temporal images at different levels of the correlation, giving an insight into the distribution of the deformation in the depth of the sample during the inclination. Figure 8 shows how we proceed. The original spatiotemporal graph is shown on Figure 8 (a). Figure 8 (b) and (c) are binarized figures obtained from Figure 8 (a) for different values of the threshold. For Figure 8 (b), all values of correlations smaller (respectively greater) than 0.15 are black (resp. white). In the case of Figure 8(c), the procedure is the same with a value of the threshold for the binarization of 0.07 . We observe that during the inclination process the level of deformation evolves as a front in the sample: the depth at which a fixed level of deformation is reached in the sample depends linearly on the angle of inclination. Figure 8(d) shows a superposition of the fronts extracted from the binarized images for five different values of the thresholds. We observe that the slope of the front remains approximately constant. The solid line Figure 8 (a) is obtained by averaging the slopes of the fronts from Figure 8 (d). This shows that the growth of the precursors events is identical to the overall repartition of the deformation in the sample. Indeed, the average slope over several experiments, all carried out in "normal" conditions of packing fraction and relative humidity, is $13 \mathrm{~d} /{ }^{\circ}$ for glass beads and $8 \mathrm{~d} /{ }^{\circ}$ for sand. Those slopes are of the same order and in the same ratio as the values of the variation of the depth of the precursors with angle $\left(9 \mathrm{~d} /{ }^{\circ}\right.$ for glass beads and $4 d /{ }^{\circ}$ for sand $)$ determined from data plotted on Figure 6 .

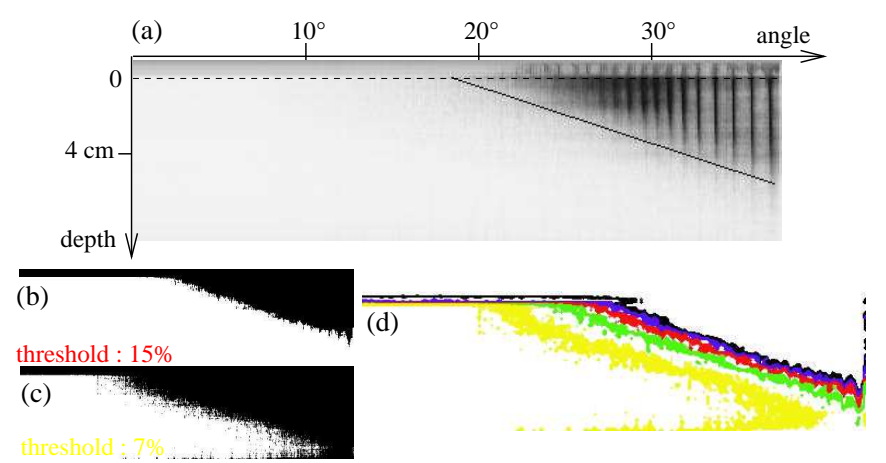

FIG. 8. (Color online) (a) Spatio-temporal representation of the $x$-averaged correlation $G_{I}$ as a function of the depth and of the inclination angle. The material is sand in "normal" conditions. The dashed line gives the mean slope of the fronts extracted from the binarized images (see main text). (b) and (c) Same data binarized with two different thresholds: (b) 0.15 and (c) 0.07. (d) Fronts extracted from the binarized spatio-temporal graphic for different values of the threshold, the gray scale is lighter when the threshold is smaller; yellow: 0.07, green: .11, red: .15, blue: .19, black: .23.

In addition to this front which shows a linear relation between angle and depth at a value of the deformation and which superimposed on the growth of the precursors at large deformations, a lot of experiments display another deformation front very close to the surface. Such a phenomenon is certainly linked to a boundary effect in 
the vicinity of the free surface. An example of such behavior is shown on Figure 9. We observe in that figure that apart from the main front that overlaps the precursors, a thin zone of deformation breaks the slope of the front near the surface.

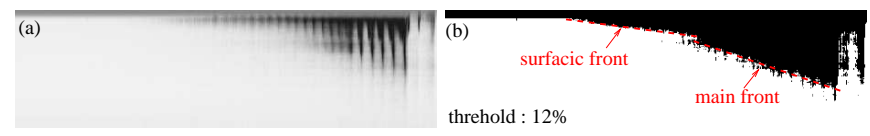

FIG. 9. (a) Spatio-temporal representation of the x-averaged correlation $G_{I}$ as a function of the depth and of the inclination angle. The material is sand in "normal" conditions. (b) Binarized image obtained from (a) for a threshold of 0.12 .

To conclude, the deformation in the sample appears as a front in a spatio-temporal representation. That front appears very soon in the inclination process and superimposed on the precursors peaks at large deformation. The linear relation that connects the angle and the depth at which the processes are observed seems to be of the same nature as the linear increase of the size of the precursors with the angle of inclination.

\section{Spots identification}

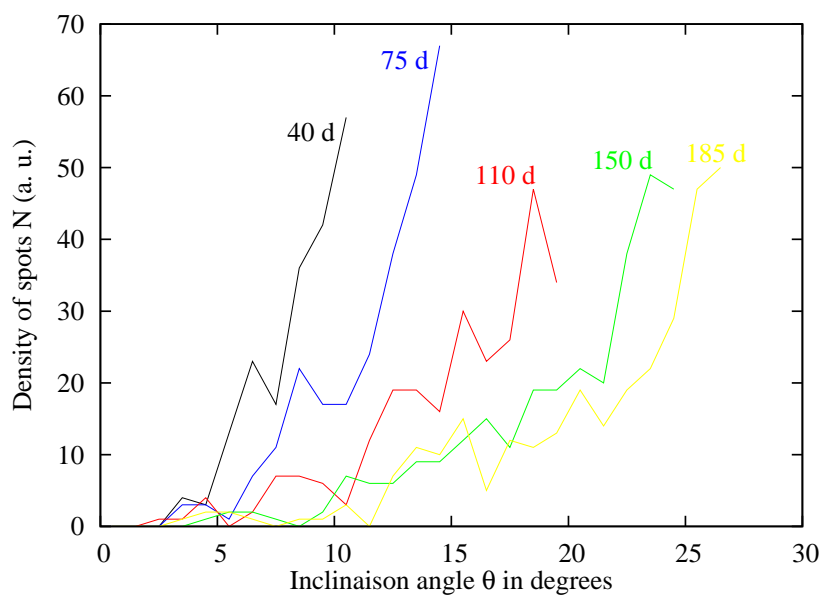

FIG. 10. (Color online) Number of spots $N$ as a function of the angle of inclination $\theta$ at different depths in the sample. The average depth (in beads diameter unity $d$ ) at which the measurement has been made is indicated near each curve. The counting has been done integrating events during $1^{\circ}$ inclination and over a slice of $\Delta z \simeq 37 d$ in depth.

Another way to quantify the activity in the material is to count the number of spots, i.e. localized deformation in the sample, and to look at its evolution during the tilt process. For this, we threshold each image in a movie, and then we identify those spots in each image. We then count the number of spots that have appeared at a certain depth during an angle increment of $1^{\circ}$. The number of spots $N$ occurring between $z-\Delta z / 2$ and $z+\Delta z / 2$, with $\Delta z$ the size of the slice, can be measured for different depths $z$, as a function of the current angle $\theta$. The result of that image analysis is given in figure 10 where the number of spots at different depths is given as a function of the angle of inclination. Each line corresponds to a different depth, from $40 d$ under the surface to $185 d$ deep. For a given depth the counting is stopped as a precursor reaching that depth occur. We observe that at a fixed depth the number of spots increases with the angle while at fixed angle it decreases with the depth. At all the depths, the density of spots increase strongly when approaching the precursor event at that depth. The final values of the densities are of the same order at all the depth.

A further analysis of those data will be done in the discussion part. The first conclusions from the raw data are that the rearrangements in the sample begin as soon as the inclination process begins, with a decreasing density in the depth of the sample. The density of spots increases strongly before the occurrence of a precursor event at the corresponding depth.

\section{Influence of parameters}

In this section, we will expose the consequences of the modification of the humidity and packing fraction on the above observations.

\section{Influence of humidity}

Figure 11 shows, for sand samples with different humidity preparations, the size of the successive precursors as a function of the angle of inclination. The experiments have been carried out with sand prepared in the same manner as previously leading to a packing fraction around .61 for the "normal" hygrometry conditions. The dispersion of the results is rather large but it can be noticed that the presence of humidity tends to increase the number of layers of grains implied in the precursory process. This is in agreement with the intuitive idea that the presence of humidity increase the cohesion of the system and consequently that more grains are involved when a rearrangement or a precursor occurs.

Other trends can also be noted: precursors appears sooner in a humid system than in a dry one. In fact, precursors are better defined in the humid case, as can be seen by comparing the spatio-temporal diagram of an experiment with a dry sample (Fig. 11(a)) and a humid sample (Fig.11(b)). In the dry case more rearrangements take place giving a blurred appearance to the diagram. The period of the precursors is also significantly smaller in the dry case.

For humid cases, a curious pairing of precursors has been observed several times, an example of which is shown in Figure 12. An oscillation between two levels 

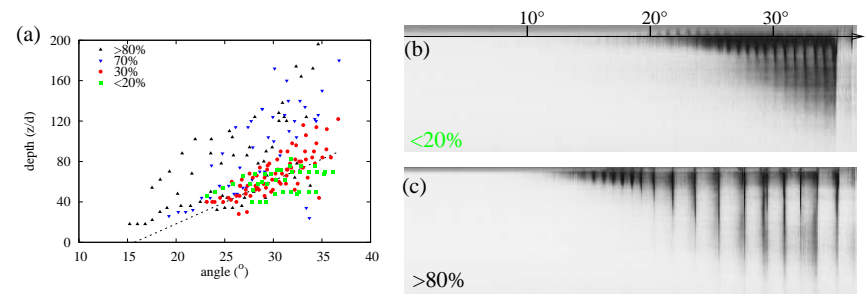

FIG. 11. (Color online) (a) Depth of the successive precursors as a function of angle for sand samples at different humidity preparation. Higher values of humidity mobilize more grain layers and the precursors begin at smaller angles. (b) and (c): Spatio-temporal diagrams of sand specimens prepared at different humidity levels. (b) Dry case (hygrometry < 20\%); (c) Humid case (hygrometry $>80 \%$ ). The packing fraction preparation of the samples is the same.

of activity can be observed, showing an alternation between two different regimes after each precursors. We also observed in some experiments an alternatation of two sizes for the precursors, which explain for example the appearance in Figure 11 of very small values of the precursors near the avalanche in some humid cases: these values alternate with much higher values.

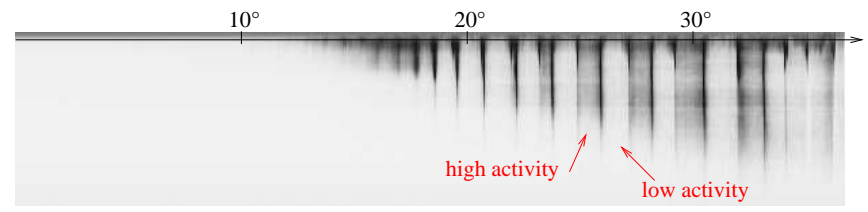

FIG. 12. Spatio-temporal diagram of sand specimen prepared at high humidity level (above $70 \%$ ): the precursors tends to appear by pairs showing a different rearrangement behavior periodically.

\section{Influence of packing fraction}

When exploring different types of packing fraction preparation, we observed that in the case of samples prepared in the loosest state, the phenomenology is the same as in the "normal" conditions. In that case the activity in the system is very high through the depth of the pile and precursors are still observed. On the contrary, the phenomenology is modified in the most compacted piles. In that case, because of the significant increase of the avalanche angle for dense materials, we were not able to reach the avalanche with our experimental setup in most of the experiments.

In the compacted experiments, local rearrangements during the inclination of the system are still observed but the activity in the system is much lower than in the less dense samples. On the contrary, large micro-failures are not always observed and when they are, they differ from the precursors events described previously. For about half of our experiments, no large events were observed at all. For the other half, large micro-slips event were observed with the main difference that the mobilized area has a clear angle with the surface of the sample instead of being parallel as was the case in the previously described micro-slips. Such a mobilized zone can be seen in Figures 13(a) to (d), where the angle of the prismatic mobilized region to the surface is about $30^{\circ}$, so that the surface of rupture is almost horizontal in the laboratory frame. The mobilized zone progresses in the system as the angle of inclination increases. This progression occurs by regular steps at a similar period to the precursors obtained in samples prepared in "normal" conditions.

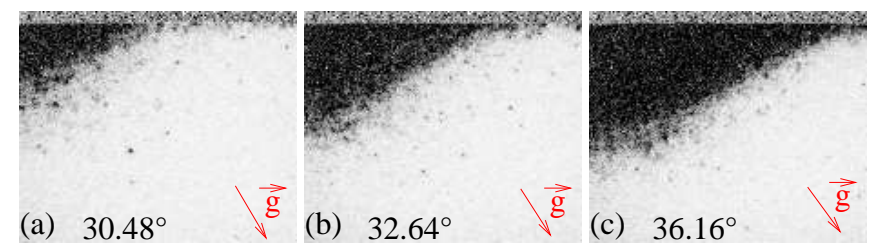

FIG. 13. Maps of correlation at different angles for a sample of sand prepared in a compacted state at "normal" hygrometry conditions (32\% relative humidity): (a) $30.48^{\circ}$, (b) $32.64^{\circ}$ and (a) $32.16^{\circ}$.

To conclude this section concerning the influence of preparation over the pre-avalanche phenomenology, we observe that the global picture of the process, i.e. the local rearrangements in the whole depth of the system and periodic micro-ruptures appearing every few degrees from an inclination of $15^{\circ}$, remains unchanged over a wide range of conditions of preparation. The phenomenon is very robust to changes in packing fraction and humidity conditions. Only the most compacted systems behave differently, presenting an internal rupture that progresses in the bulk of the system at an angle to the surface of the material.

\section{DISCUSSION}

In the preceding section, we have described the complex dynamic behavior preceding the macroscopic failure of a granular material. The dynamics begin very early in the tilting process with isolated rearrangements events. Such events have already been reported, but with our sensitive side view characterization, we are able to obtain information about their spatial distribution. At the very early stage of the tilting process the activity is essentially limited to very superficial layers, and as the tilting process progresses, the activity progresses into the bulk. A striking feature is the presence of many micro-failure events in the bulk. Such events occur quite regularly during the tilting process. We first discuss this effect in the framework of Mohr-Coulomb failure criterium. After this, we will discuss how our observations may be related to the plasticity of granular material. 


\section{A. Cohesion}

We first discuss the localization of failure in a tilted granular material as it may be deduced from Coulomb failure criteria. The equation of static equilibrium for a granular material is:

$$
\begin{aligned}
& \frac{\partial \sigma_{x x}}{\partial x}+\frac{\partial \sigma_{x z}}{\partial z}=\rho g \sin \theta \\
& \frac{\partial \sigma_{z x}}{\partial x}+\frac{\partial \sigma_{z z}}{\partial z}=\rho g \cos \theta
\end{aligned}
$$

where $\sigma_{x z}=\sigma_{z x}$ is the shear stress, $\sigma_{x x}\left(\sigma_{z z}\right)$ is the horizontal (vertical) normal stress, $\rho$ the density, and $g$ the gravity. The orientations of the axes are defined in Figure 3, and we neglect any shear stresses in the transverse $y$ direction. Assuming that the stresses are uniform in the $x$ direction, we obtain:

$$
\sigma_{z z}=\rho g z \cos \theta, \quad \sigma_{z x}=\rho g z \sin \theta
$$

The Coulomb criterion postulates that a granular material is stable with respect to failure if [34]:

$$
|\tau| \leq \mu \sigma+c_{h}
$$

for any plane inside the material. In Equation (5), $\tau$ is the shear stress, $\sigma$ the normal stress, $\mu$ the internal friction coefficient, and $c_{h}$ the cohesion. Applying the Coulomb criteria to Equation (4), we find that the planes of failure are parallel to $x$ axis, and the Coulomb criteria is now:

$$
\tan \theta \leq \mu+\frac{c_{h}}{\rho g z \cos \theta}
$$

First, in the absence of cohesion $c_{h}=0$, the depth of failure plane is not determined. With cohesion, the position of the failure plane is determined. In the case of constant cohesion, failure must occur at the bottom of the sample [35, 36], where $c_{h} / \rho g z \cos \theta$ is the lowest. That result is in contradiction with the observation that the first micro-failure appears at a smaller depth than the following ones. The dependence of $\theta$ on the values of $z$ for which failure occurs in a Mohr-Coulomb model is opposite to the dependence experimentally observed. Moreover, such a model gives only one criteria of rupture and is unable to predict successive micro-failures in the material. In any case, the Coulomb failure criterium is unable to explain the occurrence of the observed microfailures into the bulk of the sample.

\section{B. Plastic deformation}

The Coulomb failure discussed in the preceding section does not take into account any plastic deformations before rupture in the granular material. It is however well known that granular materials may yield before being subjected to failure. The critical state theory of soil 37] discussed how the plastic deformation occurs in a material depending on the initial state of the material. Starting from an initially loose granular material, application of a shear stress initially produces an elastic deformation. After this, yield begins with plastic deformations. During this yielding, the material compacts slowly. When the shear stress exceeds a threshold (generally a fraction of the confining pressure), macroscopic failure occurs in the material. If the experiment begins with an initially dense granular material, the deformation is elastic until a maximum value of the stress is reached. When the stress exceeds this value, failure occurs. No plastic deformation happens before that rupture and dilatancy occurs only after the failure. The fact that rearrangements and precursors are observed in our experiments only for loose or moderately dense granular samples (and not to densely prepared granular materials) seems to show that the observed deformations in our experiments correspond to yield before failure. We observed in a previous work, for a different experimental geometry, that the same kind of rearrangements may be associated to plastic dissipation into the material [27].

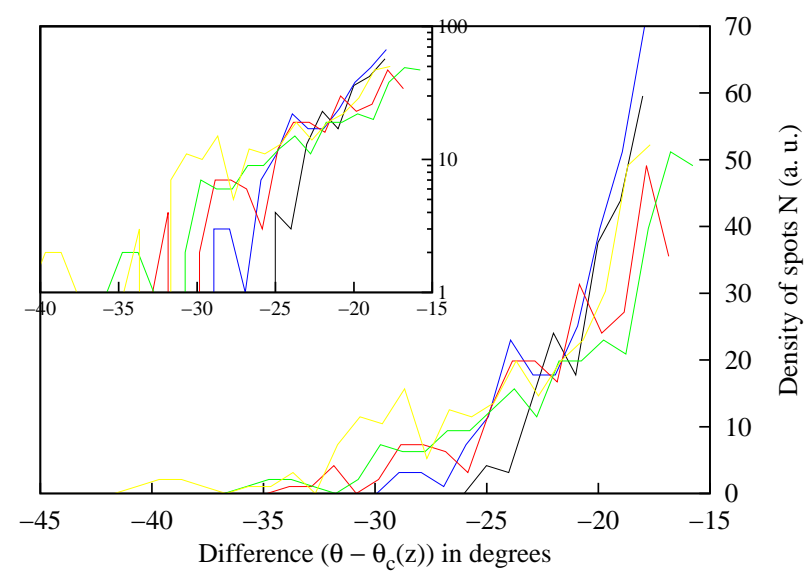

FIG. 14. (Color online) Number of spots $N$ as a function of the difference between actual angle and failure angle $\theta_{c}(z)$. Every curve corresponds to a different depth: black $z=40 d$, blue $75 d$, red $110 d$, green $150 d$, yellow $185 d$. Inset: same curves in logarithmic scale.

The link between the plastic deformations and the failure of the material may be highlighted by considering the amount of plastic deformation as a function of the difference between the applied stress and the stress at which failure occurs. We have noticed (see sec III C 1 and fig 8) that, at a given tilt angle (i.e. at a given applied stress), the density of the rearrangements decreases with the depth. This dependence of the density of the rearrangements on the depth of the material most certainly originates from a gradient of properties, either due to a depth dependent confining pressure and/or a gradient of packing fraction during the preparation of the sample. 
Such packing fraction and/or pressure dependence of the yield process is observed in many soil mechanics studies, but at confining pressures noticeably larger than the pressure in our experiment. The tilt angle at which failure occurs into the material (i.e. the failure stress) also increases with the depth (see sec IIIB2 and fig [6). From data of fig 6 , we defined $\theta_{c}(z)$ as the $z$-dependent value of the tilt angle at which failure occurs. we plot on Fig. 14 the number of events as a function of $\theta-\theta_{c}(z)$. The data obtained for different depths, which were scattered on fig 10) then collapse on the same curve. This shows that those plastic events occur with a density which is given by the difference between the failure and applied stresses, or equivalently that failures happen after a certain number of rearrangements have occurred.

It has been shown in [27] that the rate at which localised plastic events occur may be identified with the so-called fluidity of the material, which is the local rate of stress relaxation. The concept of fluidity has been introduced in order to explain the rheological properties of soft glassy material 38]. The use of this concept in order to explain rheological properties of granular material has also been recently proposed [39, 40]. The relation between isolated plastic events and failure may be qualitatively understood. Indeed, since rearrangements may be seen as plastic reorganizations, we expect that each event redistributes some stress, accordingly to an elastic stress propagator [41]. This additional stress may also trigger other plastic events in their neighborhood. Because of these processes, shear bands may form in the material [42]. It follows naturally from this kind of scenario that failure occurs when a certain amount of activity in the material is reached. This is precisely what is observed: rearrangements precede micro-failure, whatever is the depth.

\section{Periodicity of precursors}

Another striking observation is a rough periodicity in tilt angle of the precursor events. As we already shown (see sec IIIB2 and Fig. 7), those precursors events are indeed failure in the bulk material.

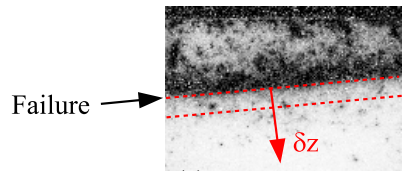

(a)

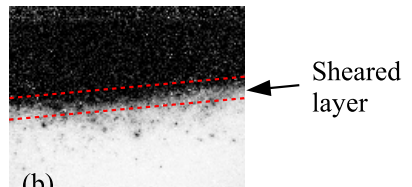

(b)
FIG. 15. (Color online) Details of a slip event. (a) is the correlation image of a microslip event showing the failure plane. (b) is the following correlation image. The zone between the dashed red lines is decorrelated although below the failure plane, and may be interpreted as a layer of sheared granular material.

We have plotted on Fig. 15] a detail of the failure already described on Fig. 7]. We see on Fig. 15] a the local- isation of the failure plane. One image latter (Fig. 15,b) we observe that the decorrelated zone extends deeper into the material. So we have between the two lines of fig 15. b a layer of material which has been deformed and which is below the failure plane. We can interpret this layer as a zone of granular material which has been sheared by the motion of the upper part. This observation is in agreement with the literature about creep motion in granular flow. It is indeed well known that when a granular layer is sheared, the deformation extends into the bulk. The deformation decays exponentially into the bulk, with a characteristic length $\xi$ of the order of few grain diameters [43 45]. The same decay in velocity is also observed during non stationary granular avalanches [46]. So the effect of a micro-failure event may be to produce a deformation which extends into the depth. This interpretation of this decorrelated layer is supported by the following quantitative analysis. Calling $\Delta$ the displacement of the upper block, we may expect [43 46] a displacement which decays as $\simeq \Delta \exp (-\delta z / \xi)$, with $\delta z$ the distance below the failure plane (see fig. 15). The deformation is then $\simeq(\Delta / \xi) \exp (-\delta z / \xi)$. With $\Delta \sim \xi \simeq d$, we have a deformation of order $10^{-6}$ (the limit of detection of our light scattering setup) for $\delta z \simeq \ln \left(10^{6}\right) \times \xi \simeq 15 d$. This is in rough agreement with the separation between the two lines of fig 15 b which is $\simeq 15 d$. This agreement supports the hypothesis that a layer of material is sheared below the failure plane.

The effect of this shear deformation may be to erase all the possible site for future micro-failure events because of the reconfiguration of the sample in that zone. The next micro-failure site should then be located slightly deeper into the bulk. The typical depth depth of this sheared layer slip being $15 d$, we may expect from the data of section III B 2 (variation of precursor depths with failure angle $\simeq 9 \mathrm{~d} / \mathrm{deg}$ for glass beads) that the angular period between successive micro-failure is of the order of $\simeq 15 / 9 \approx 1.6 \mathrm{deg}$, a value which is close to the period that we measured (see figure 5).

\section{CONCLUSION}

Our experiments on quasi-static tilting of granular materials show, in agreement with numerous previously published studies, that reorganizations occur before the avalanche takes place. The main advance of this study is to show that such rearrangements are organized spatially in a complex manner. For loose or moderately dense granular systems, we observe isolated reorganizations at low inclination, with a density decreasing slowly with the depth. As inclination increases, reorganizations occur under the form of micro-failure planes in the bulk, which are localized at increasing depths. The different localizations of the failure planes imply that the underlying physics is different from the stick-slip already observed in other granular experiments [15], where the slip plane remains the same. The micro-failures seem to occur when 
a given level of accumulated plastic deformation is attained. This holds at every depth. The case of dense granular systems seems to obey to a different underlying physics.

Those observations may be partially and qualitatively understood in the framework of yielding properties of granular material (e.g. Granta-Gravel material in [37]) before failure. At densities below the critical one, the granular material yields progressively before the failure of the material. This yielding is continuous, and associated to a compaction of the material. We may then interpret the observed plastic deformation as experimental manifestation of this yielding. Important deformations are then observed in the form of micro-failures, which, in this framework, form plastic flow in the material. The periodicity of such plastic flows may be understood if we consider collective granular flow: micro-failures involving granular matter in the bulk with a typical extension length, and the next micro-failure may occur in the next un-deformed zone. This explanation is evidently very partial and only qualitative. We do not expect that a granular model such as Granta-Gravel will apply completely or qualitatively, and it must be understood only as a starting point for future investigations.

A puzzling point is that, to our knowledge, such col- lective and regular precursors motions have not been reported in numerical simulations of tilted granular material. It follows from our experimental results that those collective events may only be observed with moderately dense and large enough numerical granular packing.

Another interesting point is that those micro-failures do not occur at the beginning of the tilt process. There is some minimum angle (i.e. some minimal shear stress) below which no micro-failures occur. To our knowledge, the existence of two finite critical shear stresses (one for micro-failure and one for the macroscopic failure) is not discussed in the literature. A complete understanding of the observation described in this paper appears as a challenging task.

\section{ACKNOWLEDGEMENTS}

We acknowledge the financial supports ANR "STABINGRAM" No. 2010-BLAN-0927-01. We thank Eric Clément, Lydéric Bocquet, Patrick Richard, Mickael Duranteau and Yves Le Gonidec for scientific discussions, Patrick Chasle for help with the image acquisition and Alain Faisant for the conception of the tilting optical board.
[1] H. M. Jaeger, C. Liu, and S. R. Nagel. Relaxation at the angle of repose. Phys. Rev. Lett., 62:40-43, Jan 1989.

[2] M. Bretz, J. B. Cunningham, P. L. Kurczynski, and F. Nori. Imaging of avalanches in granular materials. Phys. Rev. Lett., 69:2431-2434, Oct 1992.

[3] N. Nerone, M. A. Aguirre, A. Calvo, D. Bideau, and I. Ippolito. Instabilities in slowly driven granular packing. Phys. Rev. E, 67:011302, Jan 2003.

[4] S. R. Nagel. Instabilities in a sandpile. Rev. Mod. Phys., 64:321-325, Jan 1992.

[5] R. Fischer, P. Gondret, B. Perrin, and M. Rabaud. Dynamics of dry granular avalanches. Phys. Rev. E, 78:021302, Aug 2008.

[6] P. Bak, C. Tang, and K. Wiesenfeld. Self-organized criticality: An explanation of the $1 / f$ noise. Phys. Rev. Lett., 59:381-384, Jul 1987.

[7] Zaitsev, V. Yu., Richard, P., Delannay, R., Tournat, V., and Gusev, V. E. Pre-avalanche structural rearrangements in the bulk of granular medium: Experimental evidence. EPL, 83(6):64003, 2008.

[8] V. Gibiat, E. Plaza, and P. De Guibert. Acoustic emission before avalanches in granular media. ArXiv e-prints, June 2009.

[9] S. Kiesgen de Richter, G. Le Car, and R. Delannay. Dynamics of rearrangements during inclination of granular packings: the avalanche precursor regime. Journal of Statistical Mechanics: Theory and Experiment, 2012(04):P04013, 2012.

[10] L. Staron, J.-P. Vilotte, and F. Radjai. Preavalanche instabilities in a granular pile. Phys. Rev. Lett., 89:204302, Oct 2002 .
[11] L. Staron, F. Radjai, and J.-P. Vilotte. Granular microstructure and avalanche precursors. J. Stat. Mech., 2006:P07014, July 2006.

[12] Duran J. Sands, powders, and grains. Partially ordered systems. Springer, New York, 2000.

[13] J. M. Carlson, J. S. Langer, and B. E. Shaw. Dynamics of earthquake faults. Rev. Mod. Phys., 66:657-670, Apr 1994.

[14] S. Nasuno, A. Kudrolli, and J. P. Gollub. Friction in granular layers: Hysteresis and precursors. Phys. Rev. Lett., 79:949-952, Aug 1997.

[15] S. Nasuno, A. Kudrolli, A. Bak, and J. P. Gollub. Timeresolved studies of stick-slip friction in sheared granular layers. Phys. Rev. E, 58:2161-2171, Aug 1998.

[16] O. Ramos, E. Altshuler, and K. J. Måløy. Quasiperiodic events in an earthquake model. Phys. Rev. Lett., 96:098501, Mar 2006.

[17] S. M. Rubinstein, G. Cohen, and J. Fineberg. Dynamics of precursors to frictional sliding. Phys. Rev. Lett., 98:226103, Jun 2007.

[18] S. M. Rubinstein, G. Cohen, and J. Fineberg. Visualizing stickslip: experimental observations of processes governing the nucleation of frictional sliding. Journal of Physics D: Applied Physics, 42(21):214016, 2009.

[19] O. Ben-David, G. Cohen, and J. Fineberg. The dynamics of the onset of frictional slip. Science, 330(6001):211-214, 2010.

[20] S. Maegawa, A. Suzuki, and K. Nakano. Precursors of global slip in a longitudinal line contact under nonuniform normal loading. Tribology Letters, 38:313-323, 2010. 10.1007/s11249-010-9611-7. 
[21] J. Scheibert and D. K. Dysthe. Role of friction-induced torque in stick-slip motion. EPL (Europhysics Letters), 92(5):54001, 2010.

[22] J. Trømborg, J. Scheibert, D. S. Amundsen, K. Thøgersen, and A. Malthe-Sørenssen. Transition from static to kinetic friction: Insights from a $2 \mathrm{~d}$ model. Phys. Rev. Lett., 107:074301, Aug 2011.

[23] O. M. Braun, I. Barel, and M. Urbakh. Dynamics of transition from static to kinetic friction. Phys. Rev. Lett., 103:194301, Nov 2009.

[24] S. Rubinstein, I. Barel, Z. Reches, O. Braun, M. Urbakh, and J. Fineberg. Slip sequences in laboratory experiments resulting from inhomogeneous shear as analogs of earthquakes associated with a fault edge. Pure and $A p$ plied Geophysics, 168:2151-2166, 2011. 10.1007/s00024010-0239-1.

[25] D. Klaumünzer, A. Lazarev, R. Maaß, F. H. Dalla Torre, A. Vinogradov, and J. F. Löffler. Probing shear-band initiation in metallic glasses. Phys. Rev. Lett., 107:185502, Oct 2011.

[26] V. B. Nguyen, T. Darnige, A. Bruand, and E. Clement. Creep and fluidity of a real granular packing near jamming. Phys. Rev. Lett., 107:138303, Sep 2011.

[27] A. Amon, V. B. Nguyen, A. Bruand, J. Crassous, and E. Clément. Hot spots in an athermal system. Phys. Rev. Lett., 108:135502, Mar 2012.

[28] M. Erpelding, A. Amon, and J. Crassous. Diffusive wave spectroscopy applied to the spatially resolved deformation of a solid. Phys. Rev. E, 78:046104, Oct 2008.

[29] M. Erpelding, A. Amon, and J. Crassous. Mechanical response of granular media: New insights from diffusing-wave spectroscopy. EPL (Europhysics Letters), 91(1):18002, 2010.

[30] J. Crassous. Diffusive wave spectroscopy of a random close packing of spheres. Eur. Phys. J. E, 23:145-152, 2007.

[31] Linda Djaoui and Jérôme Crassous. Probing creep motion in granular materials with light scattering. Granular Matter, 7:185-190, 2005. 10.1007/s10035-005-0210-5.

[32] see movie at url.

[33] Argon A. S. Plastic deformation in metallic glasses. Acta Metall., 27:47-58, Jan 1979.

[34] Nedderman R.M. Statics and Kinematics of Granular Materials. Cambridge University Press, 1992.
[35] T. C. Halsey and A. J. Levine. How sandcastles fall. Phys. Rev. Lett., 80:3141-3144, Apr 1998.

[36] F. Restagno, L. Bocquet, and E. Charlaix. Where does a cohesive granular heap break? The European Physical Journal E: Soft Matter and Biological Physics, 14:177183, 2004. 10.1140/epje/i2004-10013-5.

[37] C. P. Schofield, A. N.; Wroth. Critical State Soil Mechanics. McGraw-Hill, 1968.

[38] C. Derec, A. Ajdari, and F. Lequeux. Rheology and aging: A simple approach. The European Physical Journal E, 4:355-361, 2001.

[39] Ken Kamrin and Georg Koval. Nonlocal constitutive relation for steady granular flow. Phys. Rev. Lett., 108:178301, Apr 2012.

[40] Pinaki Chaudhuri, Vincent Mansard, Annie Colin, and Lyderic Bocquet. Dynamical flow arrest in confined gravity driven flows of soft jammed particles. Phys. Rev. Lett., 109:036001, Jul 2012.

[41] Guillemette Picard, Armand Ajdari, Fran çois Lequeux, and Lydéric Bocquet. Slow flows of yield stress fluids: Complex spatiotemporal behavior within a simple elastoplastic model. Phys. Rev. E, 71:010501, Jan 2005.

[42] Kirsten Martens, Lyderic Bocquet, and Jean-Louis Barrat. Spontaneous formation of permanent shear bands in a mesoscopic model of flowing disordered matter. Soft Matter, 8:4197-4205, 2012.

[43] Teruhisa S. Komatsu, Shio Inagaki, Naoko Nakagawa, and Satoru Nasuno. Creep motion in a granular pile exhibiting steady surface flow. Phys. Rev. Lett., 86:17571760, Feb 2001.

[44] P. Richard, A. Valance, J.-F. Métayer, P. Sanchez, J. Crassous, M. Louge, and R. Delannay. Rheology of confined granular flows: Scale invariance, glass transition, and friction weakening. Phys. Rev. Lett., 101:248002, Dec 2008.

[45] Jérôme Crassous, Jean-François Metayer, Patrick Richard, and Claude Laroche. Experimental study of a creeping granular flow at very low velocity. Journal of Statistical Mechanics: Theory and Experiment, 2008(03):P03009, 2008.

[46] Sylvain Courrech du Pont, Raphaël Fischer, Philippe Gondret, Bernard Perrin, and Marc Rabaud. Instantaneous velocity profiles during granular avalanches. Phys. Rev. Lett., 94:048003, Feb 2005. 